\title{
Genes, environment, and caste in an ant
}

\author{
M. Breed ${ }^{1}$
}

Published online: 4 September 2015

(C) International Union for the Study of Social Insects (IUSSI) 2015

In this issue, Leniaud et al. (2015) consider the impacts of genes and environment on caste in the silver ant, Cataglyphis bombycina. The topic of genes, environment and caste continues to be a fascinating and complex arena in the study of eusocial insects, and Leniaud et al. present an exciting contribution to this discussion.

Caste is a fundamental concept in the study of eusocial insects. Differences between the reproductive caste and the worker caste often extend to morphological specializations that preclude each caste from performing the other's work. Queens can lack the tools needed for successful foraging, but have numerous ovarioles and metabolic capacity to produce large numbers of eggs. Workers of the same species then have reduced or vestigial ovaries, are missing the physical structures needed for mating, and have the morphological tools to allow them to fill the defensive and foraging needs peculiar to their species.

How are such different individuals derived in the course of development? Genes and environment, which are the two major drivers of phenotype, should explain the differences, but what is the relative role of each in caste determination? Thousands of studies of caste determination over the last century have yielded the consensus that caste is largely

M. Breed

michael.breed@colorado.edu

1 Department of Ecology and Evolutionary Biology, University of Colorado, Boulder, CO 80309-0334, USA determined by environment. The precept is that all eggs of the appropriate sex, when laid, are totipotent. This means they have equal potential to yield reproductive or worker adults. When there is more than one worker phenotype then totipotency extends to those differences as well.

But in the last two decades, we have come to appreciate that individual response thresholds to tasks can drive worker behavioral choices. These thresholds often reflect genetic differences among workers; this knowledge has brought genetics back into play in conversations about caste and task performance in eusocial insects.

Leniaud et al. take on a much different, but equally exciting, aspect of the overall question of gene/environment interactions in caste in eusocial insects. In C. bombycina, there are two distinct worker castes. One of these fits the norm for most ants-workers which are, within the caste, morphologically uniform, but variable in size. This size variance is associated with task performance.

The other worker caste in C. bombycina, though, is unusual. Soldiers are relatively invariant in size and stand as a morphologically distinct group from the workers. This contrasts with many ants in which "soldiers" are workers from the large end of the size spectrum. Soldier distinctiveness as a caste is also known in some types of termites and a few other ant species.

Leniaud et al. (2015) found that environment likely is the preponderant influence on caste determination in C. bombycina. This fits well with the consensus model of totipotency. In a few colonies, though, they found evidence for a genetic influence on soldier determination based on patrilineal effects. These results are highly important because they suggest that if we backtrack in our thinking about gene $\times$ environment interactions in caste determination, we may find other examples in which genetic 
influences, while smaller than environmental influences, are nonetheless present and important in the development of workers during caste differentiation. The results in this paper are well worth considering in the continuing search for the mechanisms of caste differentiation.

\section{References}

Leniaud L, Pearcy M, Taheri A, Aron S (2015) Testing the genetic determination of the soldier caste in the silver ant. Insect Soc 62:517-524. doi:10.1007/s00040-015-0431-5 